\title{
Food and novel objects as incentives
}

WAYNE WILSON and GEORGE TAYLOR. Stephen F. Austin State College, Nacogdoches, Tex. 75961

Two experiments were designed to test preference behavior for food and novel objects by delaying the entry of novel stimuli until rats became adapted to the apparatus. Food deprivation and food presence or absence were manipulated to show that satiated rats made more novelty selections and that deprived rats were more affected by food presence.

There are two findings concerning exploratory behavior which are relevant to this paper. First, Leckart \& Bennett (1968) suggested that food and novelty were equal in their capacity to reinforce instrumental responses when hungry male rats (hooded) were given a choice between these stimuli in an E maze. Second, Lester (1967) concluded from a Y-maze experiment with male albino rats (strain $C D$ ) that food deprivation had no consistent effect on the activity and orderliness measures of exploration.

Since Leckart and Bennett did not vary food deprivation in their investigation, the question remains as to what effect this variable will have on preference behavior when rats are given free choice to food or novel stimuli. Two experiments will be reported which are designed to accentuate preference behavior by delaying the introduction of novel objects in a $\mathrm{T}$ maze until Ss become familiar with the food location. The variables of food deprivation and the presence or absence of food will be manipulated in order to examine food and novel objects as incentives.

\section{EXPERIMENT 1}

Experiment 1 tested whether formerly deprived rats prefer a previously rewarded goal area in contrast to a location containing novel objects. Table 1 outlines the conditions for Experiment 1. Complete details of the methodology and results for both experiments can be found by consulting Taylor (1968).

Subjects were 30 Sprague-Dawley female rats reared in the laboratory and placed in individual cages at 21 days. A wooden $T$ maze, covered by glass, contained a stem $14^{1 / 2}$ in. long with each arm extended $12 \mathrm{in}$. The food area was either black or white with a food cup attached to the back wall, whereas the novel area was represented by one of three gray inserts containing four distinctive figurines of various materials. A blocking door was placed randomly at one arm so that forced-choice trials to the food location could be given in Stage 1. Free-choice trials in Stages 2 and 3 permitted Ss to view the interior of both goal areas from the choice point.

Rats were gentled and adapted to 23 -h food deprivation for seven days. Three trials were given daily to each $\mathrm{S}$ throughout the experiment with an intertrial interval of $40 \mathrm{~min}$ and a 15 -sec detention period in the goal chosen. Food incentive was 1045 -mg Noyes pellets and novel incentive was exposure to different figurine inserts but never the same insert twice in one day. The plain goal box was located randomly on each of the two arms in Stage 1 and both goal boxes were positioned randomly during Stages 2 and 3 .

Figure 1 depicts a greater sustained novelty effect for control Ss which approached significance when novel selections were summed across Days 1-8 and analyzed by a post hoc Kruskal-Wallis test $(\mathrm{H}=5.13, \mathrm{df}=2, \mathrm{p}<.10)$. Note that at least a $50 \%$ choice of the novel goal was maintained by all groups throughout Days 2-15. Fig. 1 also shows a definite shift in preference for the plain goal when food is reintroduced in Stage 3 even though all Ss are now food satiated. When compared against chance selection of the food goal across
Table 1

Design of Experiment 1

\begin{tabular}{llll}
\hline Treatment & \multicolumn{1}{c}{ Stage 1 } & \multicolumn{1}{c}{ Stage 2 } & \multicolumn{1}{c}{ Stage 3 } \\
\hline Group E & Deprived:FP & Satiated:FA & Satiated:FP \\
Group C-1 & Deprived:FA & Satiated:FA & Satiated:FP \\
Group C-2 & Deprived:FA & Deprived:FA & Satiated:FP \\
& \multicolumn{2}{c}{ Design of Experiment 2 } \\
Group 1 & Deprived:FP & Deprived:FP & Deprived:FP \\
Group 2 & Satiated:FP & Satiated:FP & Satiated:FP \\
Group 3 & Deprived:FP & Deprived:FA & Deprived:FP \\
Group 4 & Satiated:FP & Satiated:FA & Satiated:FP \\
\hline
\end{tabular}

FP refers to Food Present

$F A$ refers to Food Absent

Days 1-5 of Stage 3, a post hoc Wilcoxen Matched Pairs test was used to confirm a significant preference for the food area $(\mathrm{z}=2.7, \mathrm{~N}=28, \mathrm{p}<.05)$. It was estimated by inspection that approximately $20 \%$ of the satiated rats ate one or two pellets of the 10-pellet reward when detained in the food box. It was more typical of the animals during the latter days of Stage 2 and throughout Stage 3 to press the goal cover as if seeking release by the $\mathrm{E}$.

\section{EXPERIMENT 2}

A 2 by 2 design was proposed which permitted Ss to be food deprived or satiated throughout the experiment and allowed half the Ss continued access to food during the three stages (Table 1). Subjects were 48 female albino rats obtained from Sprague-Dawley, Inc. at 60 days of age and housed individually 30 days prior to experimentation.

The $\mathrm{T}$ maze was altered to a modified $\mathrm{E}$ maze in order to insure that Ss could not view the goal area until traversing one arm of the apparatus. Distinctive cue cards were positioned to the left and right of the choice-point area to facilitate discrimination of the two goal locations. The novel area contained one of three different inserts as before but the figurines were metallic and grounded so that tactual responses could be recorded automatically on a counter. Stage 3 was lengthened from seven to 15 days and food magnitude was reduced from 10 to two 45-mg Noyes pellets; otherwise, the procedure was administered basically as in Experiment 1. Details of the alterations and of the time measures added for Experiment 2 can be found in the complete report by Taylor (1968).

The immediate effects of forced-choice trials on the initial free choice of Stage 2 were analyzed by a chi square test based

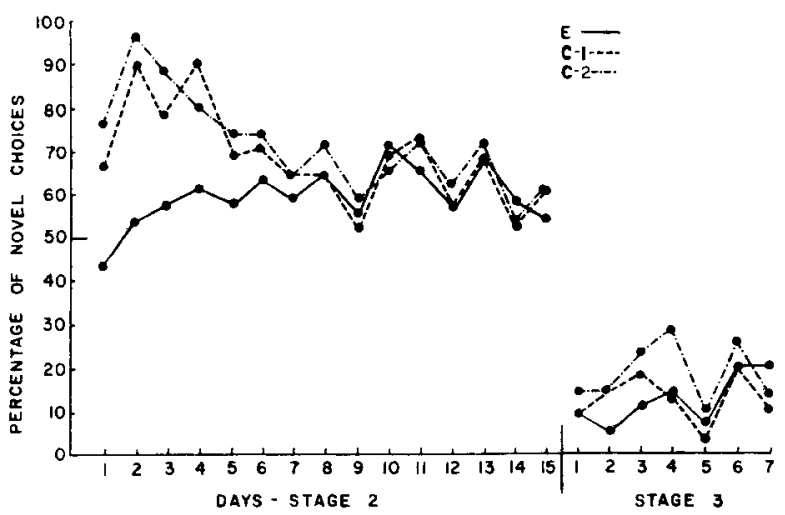

Fig. 1. The percentage of novel turns distributed across Stages 2 and 3 of Experiment 1. 
on the combined frequencies of Groups 1 and 3 and Groups 2 and 4. Results indicated that 21 of 24 deprived Ss selected the food goal but only 13 of 24 satiated Ss did so $\left(x^{2}=6.28\right.$, $\mathrm{df}=1, \mathrm{p}<.02$ ). It was decided to sum novel selections across Days 1-8 (as in Experiment 1) and study a possible interaction effect as well as the main effects produced by the food deprivation and food present-absent variables. A 2 by 2 analysis indicated greater preference for the novel goal by satiated Ss regardless of food presence or absence in the plain box $(F=84.98, d f=1 / 44, p<.01)$. Inspection of novel selections for each group during Stages 2 and 3 suggested that the food present-absent variable was of little consequence and that Groups 1 and 3 and Groups 2 and 4 could be combined without undue distortion of group trends. Fig. 2 illustrates the two curves and terminates Stage 3 after five days since the remaining 10 days presented relatively stable and redundant data. Note in both figures that the deprived Ss with food present in Stage 1 express a comparable retarded preference for novel stimuli, although the preference increases despite the fact that half the deprived $S s$ in Experiment 2 had food available during Stage 2. Also note that in Fig. 2 there is no definite shift to the food location when food is introduced in Stage 3 for half the deprived Ss and half the satiated Ss.

There were no apparent differences between satiated Ss and deprived Ss on the number of contacts with novel objects; however, this frequency did decline systematically from an average of eight contacts per $S$ on Day 1 to three contacts on Day 15 of Stage 2 . Conversely an unstable but increasing number of spontaneous alternations occurred for all groups from an estimated 19 of 40 on Day 1 to 31 of 40 possible alternations on Day 15 of Stage 2.

\section{DISCUSSION}

Despite changes in the procedure, apparatus, and preexperimental conditions of the Ss tested, Experiments 1 and 2 demonstrated a greater preference by food-satiated rats for the novel setting. The data of Experiment 1 suggested that food presence rather than food deprivation was the crucial variable since deprived Ss and satiated Ss (Groups C-2 and C-1) displayed equivalent preference behavior during Stage 2 without encountering food in Stage 1. However, food was provided for all rats in Stage 1 of Experiment 2 and then removed from half the deprived Ss and half the satiated Ss during Stage 2. Unlike Experiment 1, differences were apparent in novel selections between satiated and deprived animals indicating that food presence in Stage 1 was affecting deprived Ss more than satiated Ss.

An activity measure indexed by figurine contacts and an assessment of orderliness behavior expressed by spontaneous alternations failed to reflect differences between treatments, thereby offering support for Lester's (1967) contention that deprivation has no consistent effect on these measures of exploration. Nevertheless, Figs. 1 and 2 confirm that the deprivation variable produced a differential bias in novel selections and indicate a degree of consistency for choice behavior which should qualify as an orderliness measure.

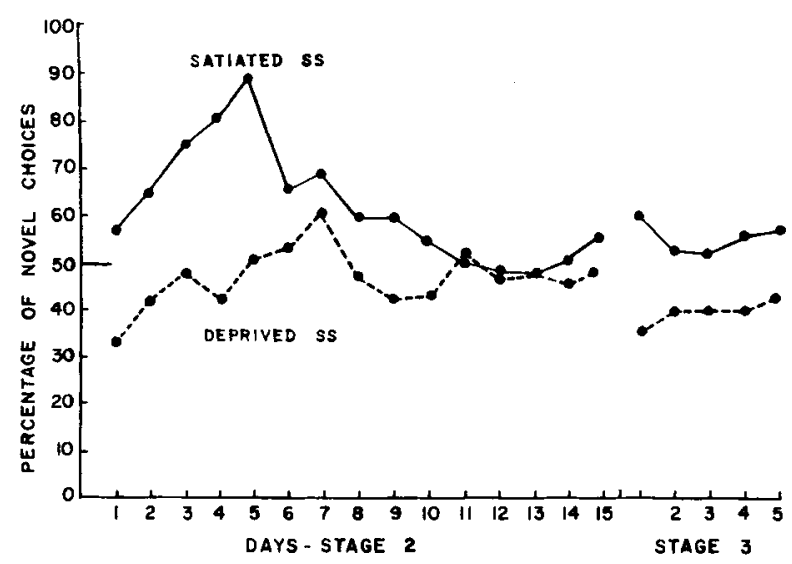

Fig. 2. The percentage of novel turns distributed across Stage 2 and five days of Stage 3 in Experiment 2.

With the exceptions noted in Figs. 1 and 2 deprived Ss and satiated Ss tended to settle into a 35 to $70 \%$ preference for the novel goal, a finding comparable to the no-preference behavior observed by Leckart \& Bennett (1968) for their food and novelty group. These authors noted that food and novelty were equal in their capacity to reinforce instrumental behavior despite the fact that their rats were food deprived but not stimulus deprived. This latter claim is questionable since their animals were maintained in individual cages (barren?) and were released daily for only one trial, a procedure which could conceivably induce stimulus deprivation by virtue of the contrast between living quarters and the test apparatus. In fact, capturing such terms as "stimulus deprivation" and "novelty" by operational definition is a difficult task. Present data revealed that as the number of figurine contacts diminished the frequency of spontaneous alternations increased, suggesting that the value of one incentive (figurines) was decreasing as the value of another incentive (?) became dominant. The food incentive is presumed to be relatively stable as long as rats are hungry but novelty values are not so durable and can be supplanted by other incentives which may or may not be associated with exploration. Thus, the relationship of preference behavior to food and novel incentives continues to be intriguing but the clarification is elusive.

\section{REFERENCES}

LECKART, B., \& BENNETT, K. Reinforcement effects of food and stimulus novelty. Psychological Record, 1968, 18, 253-260.

LESTER, D. Effects of fear upon exploratory behavior. Psychonomic Science, 1967, 9, 117-118.

TAYLOR, G. Exploratory preference behavior as a function of previously reinforced stimuli, drive level, and food presence. Unpublished Master's Thesis, Stephen F. Austin State College, 1968.

\section{(Continued from page 119)}

BLACK, R. W., \& BEVAN, W. The effect of subliminal shock upon judged intensity of weak shock. American Journal of Psychology, $1960,73,262-267$.

KRUSKAL, W. H., \& WALLIS, W. A. Use of ranks in one-criterion variance analysis. Journal of the American Statistical Association, $1952,47,583-621$.
MURRAY, H., \& KOHFELD, D. Role of AL in stimulus intensity dynamics. Psychonomic Science, 1965, 3, 439-440.

\section{NOTE}

1. This study was supported by the Air Force Office of Scientific Research, Grant No. 1163-66. 